\title{
Tear ${ }^{1} \mathrm{H}$ Nuclear Magnetic Resonance-Based Metabolomics Application to the Molecular Diagnosis of Aqueous Tear Deficiency and Meibomian Gland Dysfunction
}

\author{
José Manuel Benítez del Castilloa, ${ }^{\mathrm{b}}$ Maria Dolores Pinazo-Duran ${ }^{\mathrm{b}, \mathrm{c}, \mathrm{d}}$ \\ Silvia M. Sanz-González ${ }^{b, c, d}$ Ana M. Muñoz-Hernández ${ }^{a, b}$ \\ Jose J. Garcia-Medina b, c, d, e Vicente Zanón-Moreno ${ }^{b, c, d, f}$ \\ aDepartment of Ophthalmology, San Carlos Clinic Hospital, Madrid, Spain; 'b Spanish Net of Ophthalmic \\ Pathology (OFTARED) of the Institute of Health Carlos III, Madrid, Spain; 'Ophthalmic Research Unit "Santiago \\ Grisolía"/FISABIO, Valencia, Spain; dCellular and Molecular Ophthalmo-Biology Group, Department of Surgery \\ (Ophthalmology), Faculty of Medicine and Odontology, University of Valencia, Valencia, Spain; 'Department of \\ Ophthalmology, University Hospital Morales Meseguer, Murcia, Spain; 'International University of Valencia, Valencia, \\ Spain
}

\section{Keywords}

Meibomian gland dysfunction · Dry eyes · Aqueous tear deficiency metabolomic fingerprint - Tears · Biomarkers

\begin{abstract}
Purpose: Meibomian gland dysfunction (MGD) is a major cause of signs and symptoms related to dry eyes (DE) and eyelid inflammation. We investigated the composition of human tears by metabolomic approaches in patients with aqueous tear deficiency and MGD. Methods: Participants in this prospective, case-control pilot study were split into patients with aqueous tear deficiency and MGD (DE-MGD $[n=$ 15]) and healthy controls (CG; $n=20)$. Personal interviews, ocular surface disease index (OSDI), and ophthalmic examinations were performed. Reflex tears collected by capillarity were processed to ${ }^{1} \mathrm{H}$ nuclear magnetic resonance (NMR) spectroscopy and quantitative data analysis to identify molecules by spectra comparison to library entries of purified standards and/or unknown entities. Statistical analyses were
\end{abstract}

made by the SPSS 22.0 program. Results: Chemometric analysis and ${ }^{1} \mathrm{H}$ NMR spectra comparison revealed the presence of 60 metabolites in tears. Differentiating features were evident in the NMR spectra of the 2 clinical groups, characterized by significant upregulation of phenylalanine, glycerol, and isoleucine, and downregulation of glycoproteins, leucine, and $-\mathrm{CH}_{3}$ lipids, as compared to the $\mathrm{CG}$. The ${ }^{1} \mathrm{H}$ NMR metabolomic analyses of human tears confirmed the applicability of this platform with high predictive accuracy/reliability. Conclusions: Our key distinctive findings support that DE-MGD induces tear metabolomics profile changes. Metabolites contributing to a higher separation from the CG can presumably be used, in the foreseeable future, as DEMGD biomarkers for better managing the diagnosis and therapy of this disease.

(c) 2020 S. Karger AG, Basel

Jose M. Benítez del Castillo and Maria D. Pinazo-Durán contributed equally to this work.
Vicente Zanón-Moreno

Area of Health, Valencian International University Pintor Sorolla, 21

ES -46002 Valencia (Spain)

vczanon@universidadviu.com 


\section{Introduction}

Dry eyes (DE) result from a variety of changes occurring in 1 or more of the ocular surface system (OSS) components. In fact, dry eye is a multifactorial disease of the OS characterized by a loss of homeostasis of the tear film, and accompanied by ocular symptoms, in which tear-film instability and hyperosmolarity, OS inflammation and damage, and neurosensory abnormalities play etiological roles. Ocular discomfort and visual disability occur in varying degrees through the disease $[1,2]$. Recognized DE pathogenic mechanisms are increased tear osmolarity and OSS inflammation [3]. According to the etiology and clinical manifestations, the aqueous-deficient and evaporative $\mathrm{DE}$ forms need to be considered [4]. Main cause of evaporative DE is meibomian gland dysfunction (MGD), defined as the chronic, diffuse alteration of the gland by the occlusion of glandular orifices and/or quantitativequalitative changes in glandular secretion. The MGD may lead to tear-film changes, clinical signs/symptoms of eye irritation, inflammation, and DE (DE-MGD) [5, 6]. Studies have shown analysis-method-dependent variations in tear composition, with a wide range of metabolites identified in these biosamples [7]. Changes in metabolite availability in the OSS appear to be associated with blepharitis, $\mathrm{DE}$, or eye allergies $[5,6]$.

Metabolome is defined as the set of small molecules (i.e., intermediate metabolic products) that are present in biological tissues and fluids, the corresponding discipline being metabolomics [8]. Metabolomic profiling seeks to identify as many chemicals as possible to establish which metabolites (or metabolite patterns) discriminate samples from the cases and controls [8-10]. Metabolomic profile (MP) has become an important tool for addressing disease diagnostic/progression and for monitoring pharmacological substances [11]. High-resolution nuclear magnetic resonance (NMR) takes advantage of the magnetic properties of atomic nuclei to reveal a metabolite according to its chemical characteristics $[8,9,11,12]$. Proton NMR ( ${ }^{1} \mathrm{H}$ NMR) spectroscopic analysis of tears can provide information on the structure/composition of low-molecular-mass compounds present in a sample. This is a fast and relatively low-cost technique for exploring metabolic processes. Major advantages include unbiased metabolite detection, quantitative nature, high reproducibility, and outstanding biomedical applications [8-12], including the pioneer ophthalmic studies [13$18]$.

It might be asked whether ${ }^{1} \mathrm{H}$ NMR spectroscopy is the most appropriate analytical technique for our purposes as well as for processing our particular biosample, the human tears. Among the methods for analyzing human tears (high-pressure liquid chromatography, gas chromatography, mass spectrometry [MS], and NMR spectroscopy), the NMR may be less sensitive than MS (microgram vs. picogram, respectively) to separate/quantify the metabolites, but sufficient to detect changes in fluids. The NMR is based on the magnetic properties of some atoms, while the MS spectroscopy is based on the separation of the metabolites using chemical and physical properties. In contrast, major advantages of NMR spectroscopy include its unbiased metabolite detection, quantitative nature, and high reproducibility. Moreover, the MS needs more sample preparation and is a destructive technique $[19,20]$. Tear-film and meibum composition have been largely studied [21-23], but comprehensive tear ${ }^{1} \mathrm{H}$ NMR spectroscopy metabolomic studies in DE-MGD are lacking.

Because of the poor correlation between subjective symptoms and diagnostic DE tests, as previously stated $[1,2,22-25]$, major objective was to assess whether tear analysis - specifically analysis of the metabolome by NMR - could serve as a useful tool in the diagnosis and therapy of DE-MGD patients. We designed the present work using ${ }^{1} \mathrm{H}$ NMR spectroscopy metabolomics to analyze the capability of tear-metabolite profiling to identify metabolites that are differentially expressed in tears from the DE-MGD patient as compared to the controls.

\section{Material and Methods}

\section{Study Design}

This is a case-control study, approved by the Ethics Committee at the Hospital Clínico San Carlos (HCSC/2016; Madrid, Spain). The study fits all the principles of the Declaration of Helsinki for medical research. Risks/benefits were extensively explained, and written informed consent was signed by all subjects. We analyzed the composition of the human tears by ${ }^{1} \mathrm{H}$ NMR spectroscopy in order to determine differences in the MP that might distinguish between these 2 clinical groups: the DE-MGD patients and controls.

\section{Subjects}

According to the main inclusion/exclusion criteria (Table 1), the suitable participants were split into patients with DE-MGD $(n=15)$ and healthy controls (CG; $n=20)$. We have to emphasize that the finding of aqueous tear deficiency in many of the selected patients indicates that we have got a patient cohort with mixed pathology, if not primarily aqueous deficiency with additional MGD. Topical treatment may include only artificial tear instillation to help alleviate symptoms. Figure 1 summarizes the participants and protocols used in this study. 
Table 1. Inclusion and exclusion criteria of the study participants

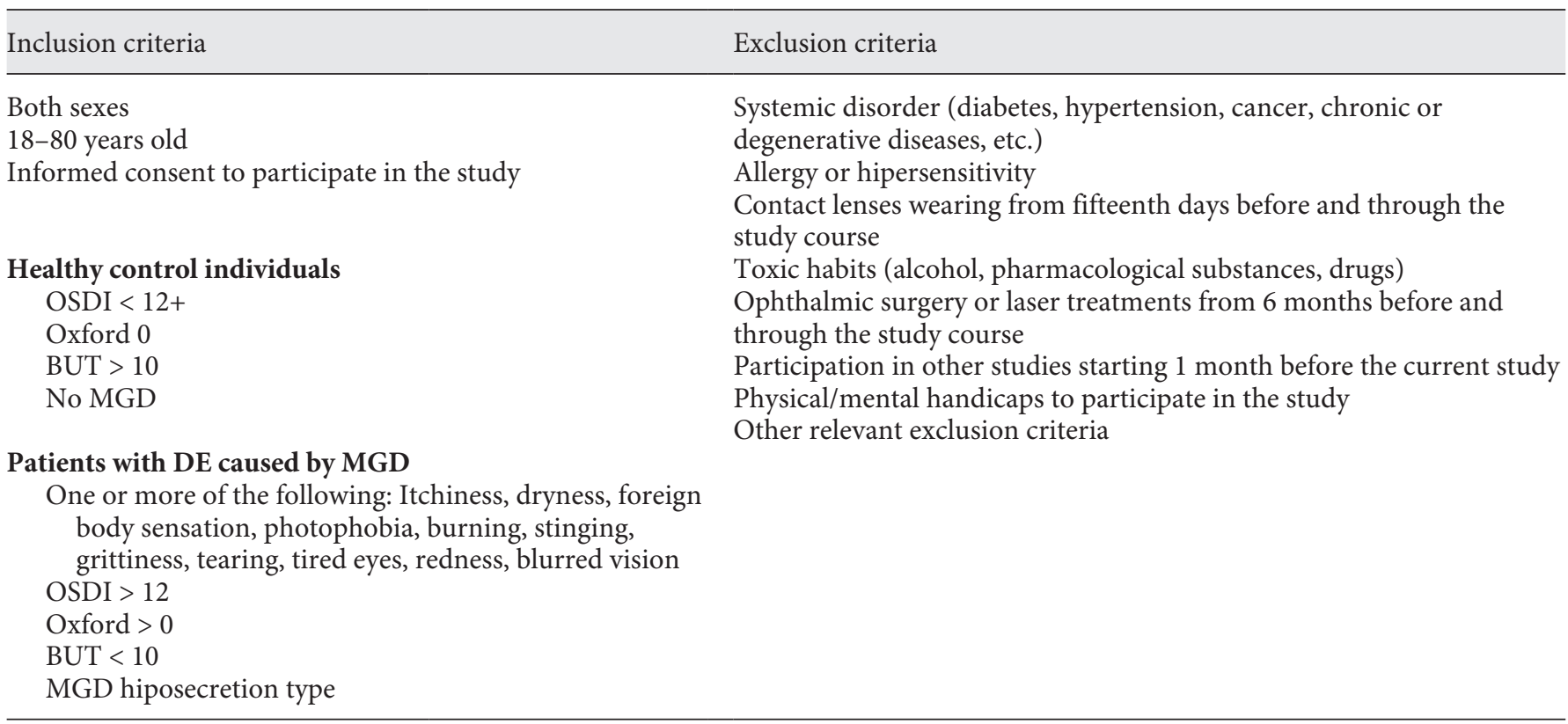

OSDI, ocular surface disease index; BUT, breakup time; DE, dry eyes; MGD, meibomian gland dysfunction.

\section{Interview and Questionnaires}

Personal interviews were performed regarding the DE signs/ symptoms and the participants' subjective sensations. The ocularsurface disorder index (OSDI [copyright held by Allergan Inc., Irvine, CA, USA]) questionnaire [26] was used in all participants for gaining information concerning the OSS symptomatology, as before $[17,18,21,22,25-27]$. Based on previous reports [28], the group of patients with symptoms of discomfort of the OSS was confirmed by its OSDI scores.

\section{Ophthalmic Examination}

A systematized eye examination was sequentially performed: (1) the best-corrected visual acuity in each eye and (2) OSS examinations using a slit lamp. The eyelid-margin morphology (irregularities, hyperemia, telangiectasias, and/or posterior displacement of mucocutaneous interface) and the MG orifices and secretion were explored (see the Fig. 2). The orifices varied from normal (in opening and appearance) to pathologic (narrowing and obstruction). The meibum expression was assessed by a semiquantitative pattern by digitally pressing on the tarsus center, observing the MG secretion (as shown in the Fig. 2), and as reported elsewhere [29]. Meibomian gland hyposecretion is characterized by decreased meibomian lipid secretion without gland obstruction. This disorder is associated clinically with gland atrophy. Diagnosis was made by (1) decreased meibum secretion after lid pressure without meibomian gland plugging and (2) meibomian gland atrophy by meibography, as previously reported [5]. Tear production was evaluated by a Schirmer test with topical anesthesia $(0.5 \%$ proparacaine hydrochloride eye drops). The strips $<10$ $\mathrm{mm} / 5 \mathrm{~min}$ were diagnosed as positive, while $\leq 5 \mathrm{~mm} / 5 \mathrm{~min}$ were diagnosed as strongly positive; OSS of both eyes was stained with $2 \mu \mathrm{L}$ of $1 \%$ fluorescein solution, which was instilled into the inferior fornix. The subject actively blinked for 3-5 s. Findings from the 4 corneal quadrants were progressively graded (no stainingto-severe staining, with confluent spots/filaments) and fluorescein breakup time (FBUT) was determined and considered positive when the final value was $\leq 10 \mathrm{~s}$. Values were the mean \pm SD of 3 examinations per everyone. Finally, the intraocular pressure was determined in each eye.

\section{Sampling Procedures}

The tear-collection method is described above. Patients were advised to stop using artificial tears at least 1-2 days before the tear collection, which was performed in the days following the baseline clinical visit. First, the eyelashes and the margins (inner/outer) of the eyelids were carefully dried with a soft towel to remove the remnant basal tears as much as possible. Then, reflex tear (the more watery type of tear produced in larger quantities whenever the eyes become gritted) was induced, this being the main tear constituent for our purposes. For reflex tear collection from all participants, the inferior meniscus of both eyes was gently rubbed with a Pasteur micro-pipette, collecting 20 - to $30-\mu \mathrm{L}$ tear samples (Fig. 3), as previously described $[17,18,20,30]$. In $16 \%$ of our cases, it was necessary to repeat the collecting procedures the following day to obtain 2 sets of tears from the same patient. Tearcollecting procedures were performed between 10:00 and 10:30 h during the corresponding appointment. Tears were transferred to Eppendorf micro test tubes. Then, a sterile plastic Pasteur pipette was attached to the top of the micro-pipette, and the rubber bulb was pressed to release the total tear volume, transferring it to the 


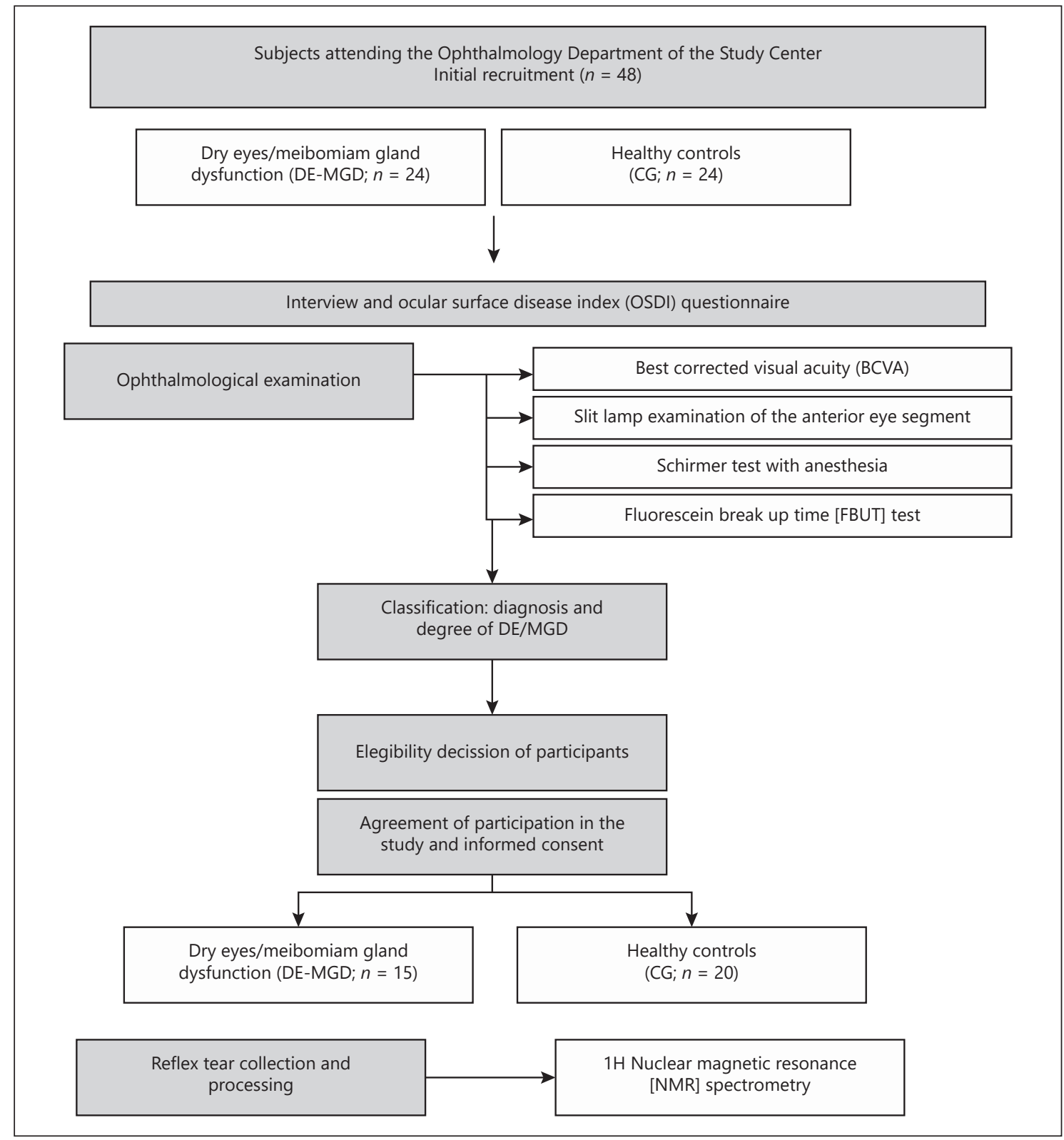

Fig. 1. Flowchart to document work-sampling procedures of the study participants. CG, healthy controls; DEMGD, dry eyes meibomian gland dysfunction; OSDI, ocular surface disease index; BCVA, best corrected visual acuity; FBUT, flourescein breakup time; NMR, nuclear magnetic resonance.

Fig. 2. The slit lamp biomicrography shows mild inflammatory signs at the lid margin, no plugs, no putting, and no capping. Diagnosis was made after lid expression that was performed in the outer region of the inner eyelid by inducing gradually the pressure with the palpebral forceps. The observer checked that nothing or scarce meibum came out from the holes of the dysfunctional meibomian glands.

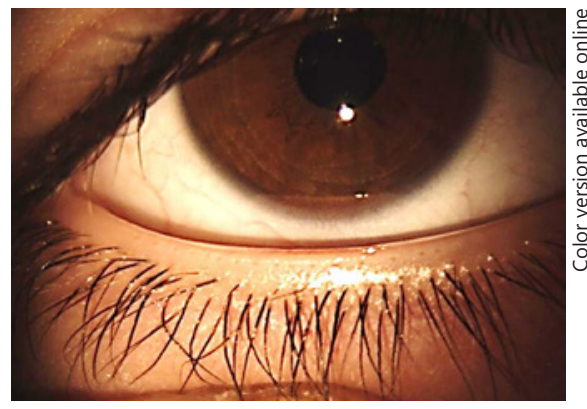


Fig. 3. Microtubes used for collecting tears and the reflex tear collection proceedings from the inner eyelid.

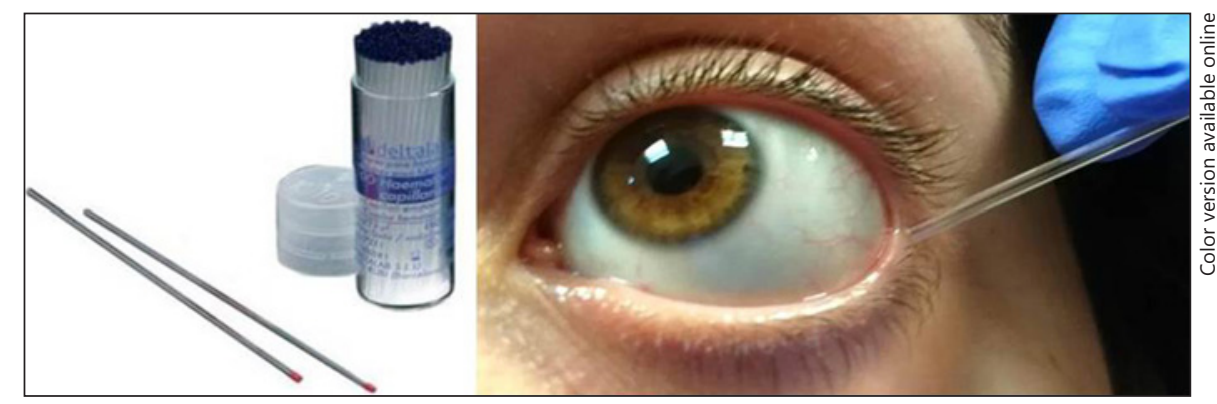

microtube. Then, tubes were marked and stored at $-80^{\circ}$ until processing.

\section{Metabolomics of Human Tears}

Tear samples were analyzed by ${ }^{1} \mathrm{H}$ NMR spectroscopy at the Faculty of Medicine and Odontology of the University of Valencia (Spain) and by the Institute of Research in Molecular Recognition and Technologic Development of the Polytechnic University of Valencia (Spain) in accordance with previous reports [13-18, 3032]. For NMR analysis, $20 \mu \mathrm{L}$ of tear was mixed with $2.5 \mu \mathrm{L}$ of 90 mM sodium-3-trimethylsilylpropionate- 2,2,3,3-d4 (TSP) in deuterium oxide. A total of $20 \mu \mathrm{L}$ of the mixture from each subject was then transferred to a high-quality 1-mm NMR tube. All ${ }^{1} \mathrm{H}$ NMR spectra were determined using a standard one-dimensional pulse sequence with water suppression on a Bruker Avance $600 \mathrm{spec}-$ trometer operating at $600.13 \mathrm{MHz}$ with a $1-\mathrm{mm} 1 \mathrm{H} / 13 \mathrm{C} / 15 \mathrm{~N}$ TXI probe. A total of 256 free induction decays were grouped into $64 \mathrm{k}$ data points with a spectral width of 14 parts per million $(\delta, \mathrm{ppm})$ and a recycle delay of $1 \mathrm{~s}$. The water signal was saturated by weak irradiation during the recycle delay. Before Fourier transformation, the free induction decay was multiplied by a $0.3 \mathrm{~Hz}$ exponential line broadening. Proton chemical shifts cover a range of 30 ppm, but the vast majority appears in the region 0-10 ppm, where the origin is the chemical shift of TSP. Therefore, spectral chemical shift referencing on the TSP signal at $0 \mathrm{ppm}$ was performed for all spectra. Tears were treated with water and strong organic solvent to separate the hydrophilic and hydrophobic compounds $[11,12]$ to achieve the proper compound isolation. Spectral regions from 0.5 to $4.4 \mathrm{ppm}$ and from 5.5 to $9.5 \mathrm{ppm}$ were binned in segments $0.01 \mathrm{ppm}$ wide $(6 \mathrm{~Hz})$ for multivariate analysis. Binned data were normalized to total aliphatic spectral area. Available spectral databases and two-dimensional (2D) NMR experiments facilitated the structural identification of the most relevant tear chemical compounds [13-15, 31, 32]. Spectra were processed using MestRenova 6.2 software (Mestrelab Research S.L., Spain) and transferred to MATLAB 7.6 (The MathWorks Inc., Natick, MA, USA) for additional processing/analysis. Signals from specific chemical compounds were integrated/quantified using semi-automated inhouse MATLAB peak-fitting routines based on Levenberg-Marquardt optimization procedures. Resolution of the NMR spectra and their signal-to-noise ratios (SNRs) (this latter defined as the ratio of signal power to the noise power which measures the original signal corruption in decibels) and the way this parameter may be corrupted during the process by undesirable noise. A higher SNR guarantees the clear acquisitions of the procedure. In this context, the resonances of the analytes present in the tear samples have been revised, and the SNRs, being low, have been validated. We only considered peaks that appear in at least $60 \%$ of the spectra reducing the chance of these peaks belonging to the noise. Resonances were assigned according to the Human Metabolome Database (http://www.hmdb.ca/) and were based on the characteristic cross-peak from previously mentioned 2D spectra, as in earlier reports with human tears $[13-15,17,18,30-32]$. The multiple variables analyzed during the metabolomic procedures make it necessary to ensure the accuracy of all obtained data [20, 33-35]. Despite the more limited sensitivity of proton NMR, the combination of the protocols for identifying the metabolites with using databases and possibly other ${ }^{1} \mathrm{H}$ NMR spectroscopy information makes possible and reproducible the assignment of the metabolites present in the tear samples.

\section{Statistical Analyses}

Chemometric statistical analyses were performed using inhouse MATLAB scripts and PLS Toolbox 6.7 (Eigenvector Research Inc., Wenatchee, WA, USA). Principal component analysis (PCA) and partial least squares discriminant analysis (PLS-DA) were applied to NMR spectra data matrix [8, 11, 32, 33, 36, 37]. Additional statistical procedures were employed. Higher values of these analyses indicated the presence of outstanding metabolites to carry out the discriminating classification. Data were processed to prepare a scatter plot combining the results from the statistics for the model variables with respect to model component scores. Data were expressed as mean \pm SD. A difference was considered significant at $p<0.05$. The statistical package for the social sciences (SPSS) (IBM Corp SPSS Statistics for windows version 22.0, Armonk, NY, USA) was used for statistics. The Mann-Whitney $U$ test and the Spearman's rank correlation coefficient (rho; SR) were applied to establish the relationship between DE-MGD clinical manifestations, the morphologic/functional DE parameters, and the tear-film metabolites. Finally, $p<0.05$ was considered statistically significant.

\section{Results}

Ocular data considered for the DE-MGD diagnosis were the baseline OSDI scores, eyelid evaluation, Schirmer, and FBUT test results (Table 2). Mean age of the study participants was $51 \pm 19$ years of the DE-MGD group and $46 \pm 12$ years of the CG. Participant distribution was $43 \%$ 
Table 2. Characteristics of the study participants

\begin{tabular}{|c|c|c|c|c|c|c|c|c|}
\hline Group & $\begin{array}{l}\text { Participant } \\
\text { distribution, \% }\end{array}$ & $\begin{array}{l}\text { Mean age, } \\
\text { years }\end{array}$ & $\begin{array}{l}\text { Gender } \\
\text { men/women, \% }\end{array}$ & OSDI scores & $\begin{array}{l}\text { MG secretion } \\
\text { semiquantitative } \\
\text { evaluation }^{\#}\end{array}$ & $\begin{array}{l}\text { Schirmer, } \\
\mathrm{mm}\end{array}$ & $\begin{array}{l}\text { FBUT, } \\
\mathrm{s}\end{array}$ & $\begin{array}{l}\text { IOP, } \\
\mathrm{mm} \mathrm{Hg}\end{array}$ \\
\hline $\mathrm{CG}(n=20)$ & $57 \%$ & $46 \pm 12$ & $54 / 46 \%$ & $4 \pm 3$ & 0 & $19 \pm 9$ & $14 \pm 3$ & $14 \pm 1$ \\
\hline
\end{tabular}

DE-MED, dry eyes meibomian gland dysfunction; CG, control group; OSDI, ocular surface disease index; FBUT, fluorescein breakup time; IOP, intraocular pressure. ${ }^{* *}$ Significant differences $(p \leq 0.001) .{ }^{\#}$ Grading from absent to hypersecretion.

in the DE-MGD patient versus $57 \%$ of the CG. Regarding gender (men/women), our participants were distributed into $66 / 34 \%$ of the DE-MGD patient and $54 / 46 \%$ of the CG.

The DE-MGD patient reported a history of dryness before this study, while the CG showed no DE-related signs/symptoms. Moreover, it has also to be mentioned that some DE-MGD patients displayed very low Schirmer test data, as reflected in the Table 2 , indicating the presence of aquodeficiency DE according to the TFOS-DEWS II report [2]. The Figure 2 shows a slit lamp image of hyposecretory non-obstructive MGD. None of the patients suffered from Sjögren's syndrome.

In both, the DE-MGD (SR: $0.745[p=0.001]$ and 0.712 $[p=0.003]$, respectively) and the CG (SR: 0.825 [ $p=$ $0.000008]$ and 0.860 [ $p=0.000001]$, respectively), total tear lipids correlated positively and significantly with the Schirmer and the FBUT tests.

To build the tear metabolomic fingerprint, we first constructed the CG tear metabolome and next assigned the DE-MGD tear resonances. Tear-metabolite concentrations were within the millimolar range, which are several orders of magnitude lower than the water concentration. Therefore, ${ }^{1} \mathrm{H}$ NMR spectroscopic imaging needs the pre-saturation of the abundant water signals. Sixty tear chemical compounds were detected. Several unidentified compounds were excluded from our results (unknown peaks: UP-01 to UP-06 from the aliphatic region and UP-07 to UP-09 from the aromatic region of the same ${ }^{1} \mathrm{H}$ NMR spectral comparisons), 51 chemical compounds were properly identified in reflex tears (Table 3 ). The set of tear compounds found in both groups proved to be composed mainly of lipids and amino acids (aa), the latter being the most abundant. Figure 4 shows the sum of all spectra.

The highest concentrations from the controls corresponded to glycoproteins (acetyls), leucine, mobile lipids
$\left(-\mathrm{CH}_{2}-\right)$, lipid/cholesterol, isoleucine, lipids (LDL/ VLDL), sphyngomyelin, glutamic acid/glutamate, and glycerol. Other compounds identified included several lipids signals $\left(-\mathrm{CH}_{3}\right.$ lipid and, $-\mathrm{CH}_{2}$ lipids), phospholipids, phenylalanine, valine, citrate, lactate, tryptophan, and glucose. Studying the aliphatic (0.5-4.7 ppm) and aromatic (5-10 ppm) regions, we assigned metabolites to each (Table 3).

Accurate comparison of tear compounds identified in both groups indicated that phenylalanine, glycerol, and isoleucine were upregulated, whereas glycoproteins, leucine, and $-\mathrm{CH}_{3}$ lipids were downregulated in tears from the DE-MGD patients. Phenylalanine, isoleucine, arginine, cysteine, valine, and tryptophan reached significantly higher levels, whereas glycine and leucine registered significantly lower levels in tears from the DE-MGD patients as compared to the CG. Within the carbohydrate compounds, glucose was identified as significantly higher in the DE-MGD tears than in the CG (Table 3). A total of $10 \mathrm{lipid} /$ protein compounds were identified in tears from both groups (mobile lipids, $-\mathrm{CH}_{3}$ lipids, lipid/protein, lipid/cholesterol, - $\left[\mathrm{CH}_{2}\right]_{n}$-lipids, sphyngomyelins, glycoproteins, phospholipid, phosphoethanolamine, and glycerol). The most abundant molecule found in normal tears was glycoprotein $(0.1699 \pm 0.01)$ but significantly lower values were detected in the DE-MGD patient $(0.1503 \pm$ 0.009). Regarding the organic acid derivatives, acetate, lactate, creatine, 2-methyl-oxovalerate, pyruvate, citrate, choline, amino- $n$-butyrate, and 4 -aminohippurate were found in tears from both groups of participants. However, only amino- $n$-butyrate significantly increased in concentration, while acetate significantly decreased in tear samples from the DE-MGD patient with respect to the CG. Main metabolites contributing to a wider separation between groups were monitored: phenylalanine, glycerol, isoleucine, glycoproteins, mobile lipids, leucine, $-\mathrm{CH}_{3}$ lipids, and lipid/cholesterol, as reflected in the Figure 5. 
Table 3. Comparison of "in vivo" tear metabolite ratios (absolute concentrations) between the DE-MGD versus the CG

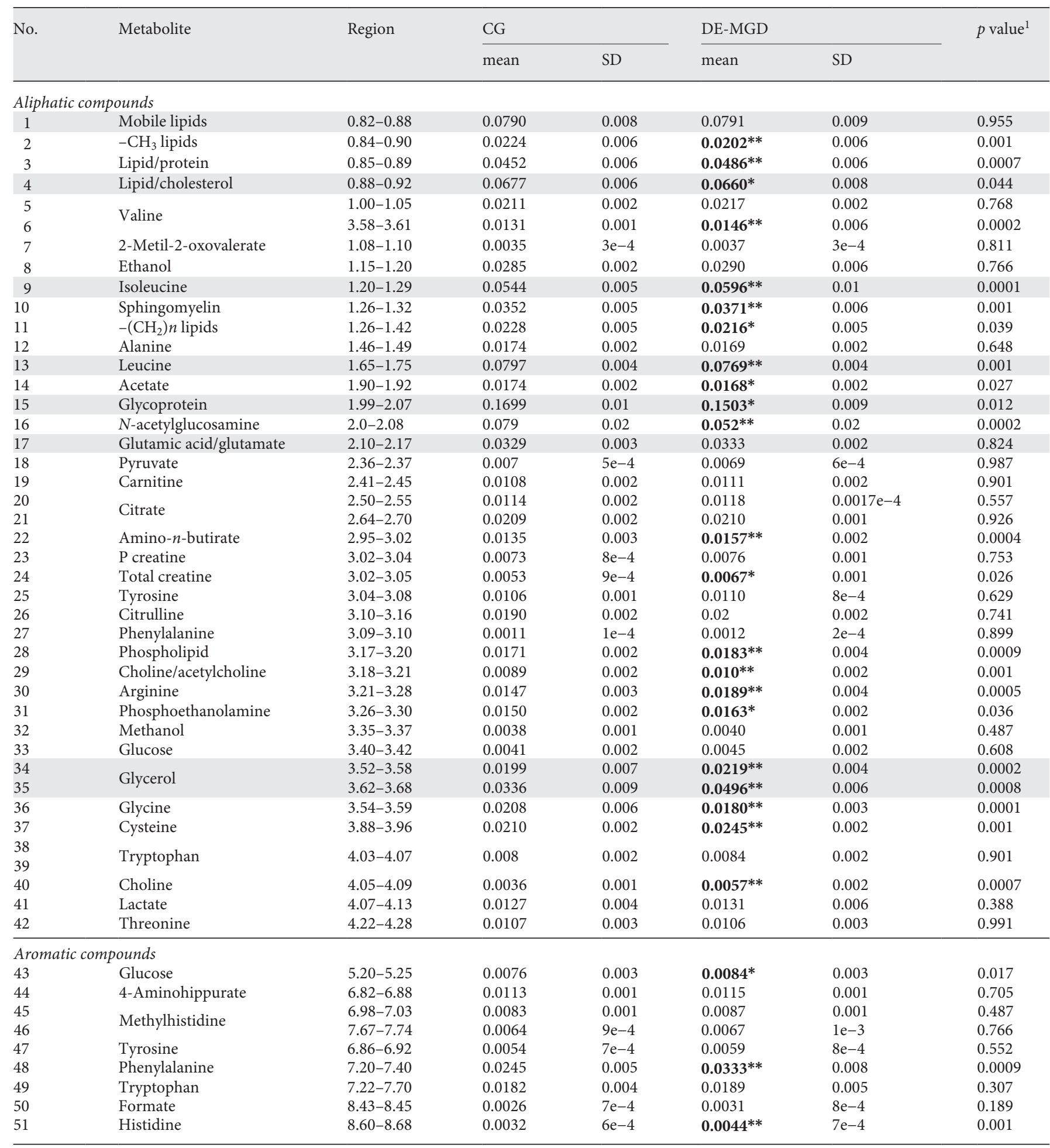

Marked in grey are the most abundant tear metabolites. CG, control group; DE-MGD, dry eyes meibomian gland disfunction; SD, standard deviation. ${ }^{1} p$ obtained from the Student's $t$ Test for independent samples. 


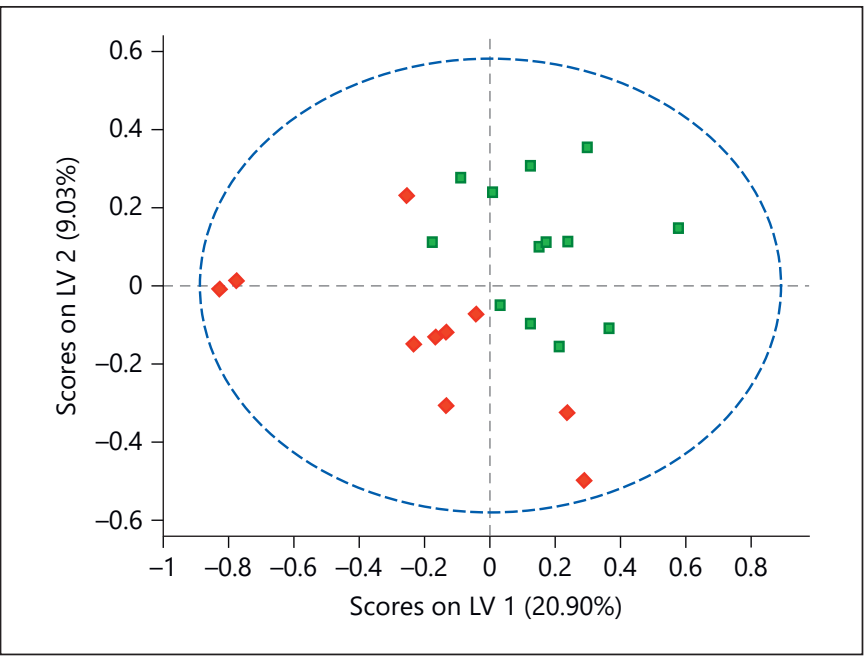

Fig. 4. PCA and PLS-DA were applied to NMR spectra data matrix. The figure shows the PLS diagram with the assigned metabolic characteristics of the human tears from the DE caused by MGD and the CG. Red figures correspond to tears from the CG. Green squares correspond to tears from the DE-MGD patients. It is recommended to use LVs for the Y matrix to carry the variance of interest from a quantitative and reproducible analytical tool. It is also suggested that a limited number of LVs be used for the Y matrix in the regression, and they should carry less than the total variation in the corresponding spectra data. DE-MED, dry eyes meibomian gland dysfunction; NMR, nuclear magnetic resonance; PCA, principal component analysis; PLS-DA, partial least squares discriminant analysis; LVs, latent variables.

\section{Discussion}

This is the first study available dealing with the qualitative/quantitative characteristics of the metabolome in tears from DE-MGD patients. ${ }^{1} \mathrm{H}$ NMR metabolomics spectroscopy was used for these approaches. Our DEMGD prediction model by using clinical measurements and tear metabolomics data revealed molecular fingerprints closely related to the OSS. Moreover, when clustering DE-MGD patients, a different MP of the healthy control subjects was detected. Because of this, the subjective symptoms assessed by personal interviews and questionnaires as well as the data gathered from the OSS clinical examination $[1,3,5,24-27,29,38]$ established the diagnosis of the DE-MGD, and the metabolomics evaluation confirmed the disease in terms of tear composition (related to OSS) (as reflected in Tables 2 and 3).

Exhaustive metabolomics data set corresponds to the estimated production of a metabolic system. The immense number of metabolic compounds makes it difficult to correctly decipher, assign, and biomedically inter- pret the information provided by the specific sample processing [36]. There is a lack of consensus among the various works published on the tear-collection procedure $[4,7,13,15,17,18,30,39-41]$. Here we collected the reflex tear samples by gentle rubbing and mild stimulation through capillarity from the inferior lachrymal meniscus of both eyes, as shown in Figure 3, and described elsewhere $[17,18,30]$. Authors described the effects of the reflex tear collection technique in detecting antioxidants by comparing the capillary-tube and the Schirmer-strip collection methods, and the less-invasive capillary-tube collection method was proposed as the choice for biochemical studies based on reflex tears [42], as we performed here.

The NMR spectroscopic approaches are rapid and reproducible, offering a standardized (relatively easy) technique for sample preparation and thus ensuring sample stability. When complemented with the principal component analysis, NMR spectroscopy can help to identify variations in cell metabolism in response to different stressors [43]. Our data from the tear chemometric analysis and ${ }^{1} \mathrm{H}$ NMR spectra comparison revealed the presence of 60 chemical compounds. Nine molecules (unidentified peaks: UP-01 to UP-09) were unable to establish proper identification with the Human Metabolome Database [44]. According to the literature search, the excellent analytical reproducibility and robustness of ${ }^{1} \mathrm{H}$ NMR metabolomic techniques with implications for the quantitative interpretation of metabolites in human tears $[8-18,31,32,45,46]$ has been proved herein.

Figure 4 shows the PSL diagram with the assigned metabolomic fingerprint of the human tears from the DE caused by MGD and the CG. We can see how both groups are almost perfectly differentiated in this diagram. We were able to detect and perfectly identify chemical compounds in the tear samples; among them, 42 pertained to the aliphatic $(0.5-4.7 \mathrm{ppm})$ and 9 to the aromatic regions. Most abundant compounds found in the CG tears were glycoproteins, leucine, mobile lipids, lipids/cholesterol, isoleucine, lipids/proteins, and $-\mathrm{CH}_{2}$ lipids. Studying the aromatic region (5-10 ppm), we identified glucose, 4-aminohippurate, methylhistidine, tyrosine, phenylalanine, tryptophan, formate, and histidine. All the data gathered enabled us to build the human tear metabolome (Table 3).

By additional statistical procedures, as described above, we selected compounds that contributed heavily to the separation between groups (as shown in the Fig. 5). Six discriminant variables, 3 upregulated (phenylalanine, glycerol, and isoleucine), and 3 downregulated (glyco- 
Fig. 5. The figure represents the sum of all spectra obtained from the tear-film metabolomics that are shown in a separate fashion. Representative spectra for the chemical standards for major metabolites (a), the control tears (b), the DE-MGD tears. The "lipids" identified in the spectra correspond to mobile lipids, $-\mathrm{CH}_{3}$ lipids, lipid/ protein and lipid/cholesterol regions (c). Finally, main metabolites contributing to a wider separation between groups in the PLS-DA diagram were phenylalanine, glycerol, isoleucine, glycoproteins, mobile lipids, leucine, $-\mathrm{CH}_{3}$ lipids, and lipid/cholesterol. DE-MGD, dry eyes meibomian gland dysfunction; PLS-DA, partial least squares discriminant analysis.
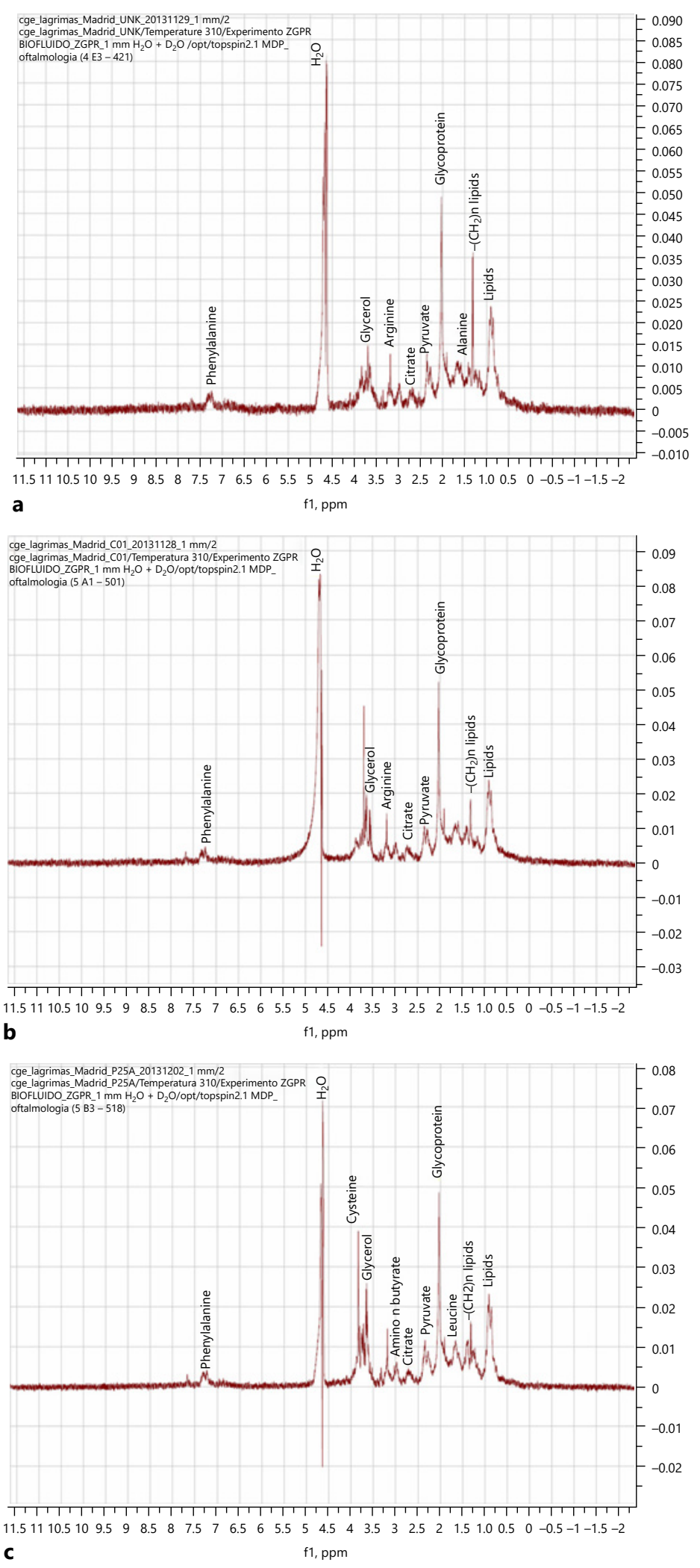

c 
proteins, leucine, and $-\mathrm{CH}_{3}$ lipids) compounds were found. The above substances underwent statistically significant changes, suggesting the existence of differential metabolomic fingerprints for tears from the DE-MGD group with respect to the CG. Owing to the complexity of the tear metabolome, the main results of our study are discussed separately.

It has been reported that human tears contain 23 aa and that the availability and functionality of these may be altered by pathologic OSS processes [25]. Notably these authors also reported that aa profiles of the basal- and reflex tears gave similar results. Some free aa have been used to treat OSS disorders $[47,48]$.

We may suggest that some of the identified aa may play significant roles in carbohydrate synthesis in healthy tears. The observed tear upregulation of phenylalanine, isoleucine, arginine, cysteine, choline, valine, and tryptophan in the DE-MGD patients may be explained by the essential functions of these metabolites in several signaling pathways of OSS pathologies. It is also plausible that a major process of releasing the above aa into tears could occur in order to convert these molecules into others. Moreover, phenylalanine overexpression in the DE-MGD tears may reflect the proinflammatory response, as suggested elsewhere $[4,17$, $18,49]$. It has been previously reported that reflex tears contain several antioxidants, including cysteine, ascorbate, glutathione, urate, and tyrosine in addition to other molecules [42]. Also, arginine has been proposed to modulate T-cell metabolism and cell survival via gene expression [50]. We detected noticeably higher levels of phenylalanine, arginine, cysteine, tyrosine, and methylhistidine in the DE-MGD tears. These results may be useful to address OSS metabolic dysfunction as well as inflammatory and oxidative stress processes occurring in the DE-MGD eyes.

A mild increment of glucose was detected in the DEMGD tears. It has been recently reported that $1 / 2$ to $2 / 3$ of diabetics suffer DE with the characteristic that the higher the Hb1AC values, the higher the severity of the OSS disease. In fact, there is a significant increase in the appearance of MGD in diabetics as compared to non-diabetics [51]. The precise levels of glucose in the tear-film from diabetics (and controls) remain controversial [52].

As widely recognized, meibum is a complex mixture of various lipids that are essential for the functionality of the tear-film/OSS integrity. Composition of the human tear-film and meibum includes hydrocarbons, wax, and cholesteryl esters and cholesterol, in addition to triacyl and diacyl glycerols, phospholipids, sphingomyelin, O- acyl-omega-hydroxy fatty acids, and a variety of other molecules [53-62]. Studies using high-performance thinlayer chromatography semiquantitative methods of human tears reported an average global tear-lipid concentration of $0.13 \mathrm{~g}$ of lipids/L [59], in contrast to a later report of a total tear-lipid concentration of $1.8 \pm 0.8 \mathrm{~g} / \mathrm{L}$ [61], the most noteworthy being free cholesterol, free fatty acids, triacylglycerols, and polar lipids. Several lipidassociated signals are visible in ${ }^{1} \mathrm{H}$ NMR spectroscopy, such as the methyl $\left(-\mathrm{CH}_{2}-\right)$ signal at $0.84-0.90 \mathrm{ppm}$ and the methyl $\left(-\mathrm{CH}_{3}\right)$ signal at $1.26-1.42 \mathrm{ppm}$. Among the lipid compounds found in tears of the DE-MGD study group were mobile lipids, $-\mathrm{CH}_{3}$ lipids, lipid/protein, lip$\mathrm{id} /$ cholesterol, sphyngomyelins, $-\left(\mathrm{CH}_{2}\right)_{n}$ lipids, glycoprotein, phospholipid, phosphoethanolamine, and glycerol. Cellular lipid levels are affected by different stressors $[47,53,54,62]$.

It has recently been reported that sebum depletion from mouse-eye MGs negatively influenced the ability to repel water and increased the susceptibility to UVB-induced apoptosis [63]. Another noteworthy point in our findings was the presence of lipids/cholesterol in tears at $0.88-0.92 \mathrm{ppm}$, in agreement with previous reports pointing to cholesterol (and/or epi-cholesterol) as the most abundant sterol in the MGs lipids together with O-acylomega-hydroxy fatty acids [64]. We found sphingomyelins in tears, as well as phospholipids [54], suggesting that changes in uptake, utilization, and maintenance of these metabolites may help alter the tear-film composition of the DE-MGD patient. In this context, it has been reported [63] that tear-film instability can be considered the key point of DE disorders. It has been also speculated [64] that different tear-film components (meibomian lipids, ocular mucins, and proteins) may influence tear-film instability. Moreover, it was said [65] that MGD patients may have different compositions of meibomian lipids, specifically considering that the lipid profile could be an important indicator to diagnose the disease. It has also been conjectured that MGD is the result of a deficiency in meibum and/or abnormal lipids to constitute the tearfilm lipid layer, leading to reduced tear-film stability [66]. In view of all scientific evidence on the role of lipids in the MGD [21, 22, 67, 68], and the differential MP found in DE-MGD tears, our data demonstrate that among the 8 tear compounds contributing to a sharper separation between the 2 study groups, 5 of these $(62 \%)$ were lipids or were related to lipids (glycerol, mobile lipids, $-\mathrm{CH}_{3}$ lipids, and cholesterol lipids). The role of other tear molecules found during the metabolomic approaches (2- methyl2 -oxovalerate, involving isoleucine biosynthesis; 4 -ami- 
nohippurate, an amide derivative of the carboxylic hippuric acid; 2 methylhistidine, an index of protein degradation) requires further research.

Current understanding of OSS at the cellular level has been noticeably enhanced by HR images from minimally invasive emerging technologies, such as the in vivo confocal scanning laser microscopy $[68,69]$ or the spectral-domain optical coherence tomography infrared meibography [70]. To determine the links between OSS clinical changes and molecular information, new challenging assays by analyzing human tears have arisen. Metabolomics of human tears using small sample volumes [8-18, 31, 32, $45,71]$ offers a different level of information concerning the biological processes occurring in the OSS that are difficult to gain through other omics platforms. By maximizing the tear collecting and handling procedures, the chemical compounds extraction and SNR efficiency, we have evolved an optimized protocol for ${ }^{1} \mathrm{H}$ NMR human tear metabolomic analysis applicable to dry eye studies caused by MGD.

Regarding the study limitations, the following should be taken into consideration: (1) the relatively small number of participants (35 subjects) would benefit from the use of a larger sample in our future DE-MGD metabolomics research; (2) our preliminary results do not intend to be conclusive. But the possibility that the compounds contributing to a wider separation between the 2 study groups can be used as diagnostic biomarkers of the DEMGD process should be validated through further research; (3) in general the biomedical potential of metabolomics is likewise hampered by the noticeable deviation in interpretation confidence; and (4) the small differences found between tear metabolites strongly suggest that the data will improve when validated by omic approaches at the protein level.

\section{Conclusions}

In view of the differential MP found in tears of both groups, we suggest that the most useful compounds for classification are phenylalanine, glycerol, isoleucine, glycoproteins, mobile lipids, leucine, $-\mathrm{CH}_{3}$ lipids, and lipid/ cholesterol. These may be considered candidate molecules to be further validated and used for the diagnosis in this particular defined group, the DE-MGD patient.

\section{Acknowledgement}

The authors would like to thank to M.C. Matínez-Bisbal and R. Martínez-Mañes from the Interuniversity Institute of Research in Molecular Recognition and Technologic Development of the Polytechnic University of Valencia (Spain) for excellent assistance and critical comments.

\section{Statement of Ethics}

The studies involving human participants were reviewed and approved by CEIC University Hospital San Carlos (HCSC/2016; Madrid, Spain). The patients/participants provided their written informed consent to participate in this study.

\section{Conflict of Interest Statement}

The authors declare that the research was conducted in the absence of any commercial or financial relationships that could be construed as a potential conflict of interest.

\section{Funding Sources}

This work is the result of the collaboration of members assigned to the nationwide research teams of the RETICs Ophthalmology Network: OFTARED of the Health Institute Carlos III (Spanish Ministry of Economy, Industry and Competitiveness). This work has been also funded, in part, by the European Program FEDER and by the BIOMEGA-UPV/FISABIO research Project (2018-2019).

\section{Author Contributions}

Benítez-del-Castillo and Pinazo-Durán share the first place within the authors. They both designed, recruited patients, obtained biosamples, obtained fundus, wrote, and edited; Sanz-González did much laboratory work, wrote, and edited; Muñoz-Hernández recruited patients and obtained biosamples; García-Medina recruited participants and obtained biosamples, wrote; Zanón-Moreno designed, wrote, edited, and reviewed the manuscript.

\section{References}

1 The definition and classification of dry eye disease: report of the Definition and Classification Subcommittee of the International Dry Eye WorkShop (2007). Ocul Surf Surf. 2007 Apr;5(2):75-92.
2 Craig JP, Nichols KK, Akpek EK, Caffery B, Dua HS, Joo CK, et al. TFOS DEWS II definition and classification report. Ocul Surf. 2017; 15(3):276-83.
3 Lemp MA, Bron AJ, Baudouin C, Benítez Del Castillo JM, Geffen D, Tauber J, et al. Tear osmolarity in the diagnosis and management of dry eye disease. Am J Ophthalmol. 2011 May 01;151(5):792-e1. 
4 Enriquez-de-Salamanca A, Castellanos E, Stern ME, Fernandez I, Carreno E, GarciaVazquez C, et al. Tear cytokine and chemokine analysis and clinical correlations in evaporative-type dry eye disease. Mol. Vis.. 2010 May 19;16:862-73.

5 Nelson JD, Shimazaki J, Benitez-del-Castillo JM, Craig JP, McCulley JP, Den S, et al. The international workshop on meibomian gland dysfunction: report of the definition and classification subcommittee. Invest Ophthalmol Vis Sci. 2011 Mar 30;52(4):1930-7.

6 Gipson IK. Age-related changes and diseases of the ocular surface and cornea. Invest Ophthalmol Vis Sci. 2013 Dec 13;54(14):ORSF4853.

7 Zhou L, Beuerman RW. Tear analysis in ocular surface diseases. Prog Retin Eye Res. 2012 Nov;31(6):527-50.

8 Lindon JC, Holmes E, Bollard ME, Stanley EG, Nicholson JK. Metabonomics technologies and their applications in physiological monitoring, drug safety assessment and disease diagnosis. Biomarkers. 2004 February 01; $9(1): 1-31$.

9 Bodi V, Sanchis J, Morales JM, Marrachelli VG, Nunez J, Forteza MJ, et al. Metabolomic profile of human myocardial ischemia by nuclear magnetic resonance spectroscopy of peripheral blood serum: a translational study based on transient coronary occlusion models. J Am Coll Cardiol. 2012 May 1;59(18): 1629-41.

10 Deidda M, Piras C, Binaghi G, Congia D, Pani A, Boi A, et al. Metabolomic fingerprint of coronary blood in STEMI patients depends on the ischemic time and inflammatory state. Sci Rep. 2019 Jan;9(1):312-y.

11 Weljie AM, Newton J, Mercier P, Carlson E, Slupsky CM. Targeted profiling: quantitative analysis of $1 \mathrm{H}$ NMR metabolomics data. Anal Chem. 2006 Jul 1;78(13):4430-42.

12 Trygg J, Holmes E, Lundstedt T. Chemometrics in metabonomics. J Proteome Res. 2007 Feb;6(2):469-79.

13 Young SP, Wallace GR. Metabolomic analysis of human disease and its application to the eye. J Ocul Biol Dis Infor. 2009 Nov;2(4):23542.

14 Midelfart A. Metabonomics--: a new approach in ophthalmology. Acta Ophthalmol. 2009 Nov;87(7):697-703.

15 Chen L, Zhou L, Chan EC, Neo J, Beuerman RW. Characterization of the human tear metabolome by LC-MS/MS. J Proteome Res. 2011 October;10(10):4876-82.

16 Agudo-Barriuso M, Lahoz A, Nadal-Nicolás FM, Sobrado-Calvo P, Piquer-Gil M, DíazLlopis M, et al. Metabolomic changes in the rat retina after optic nerve crush. Invest Ophthalmol Vis Sci. 2013 Jun 21;54(6):4249-59.

17 Galbis-Estrada C, Martinez-Castillo S, Morales JM, Vivar-Llopis B, Monleón D, DíazLlopis $\mathrm{M}$, et al. Differential effects of dry eye disorders on metabolomic profile by $1 \mathrm{H} \mathrm{nu}-$ clear magnetic resonance spectroscopy. Biomed Res Int. 2014;2014:542549.
18 Galbis-Estrada C, Pinazo-Durán MD, Martínez-Castillo S, Morales JM, Monleón D, Zanon-Moreno V. A metabolomic approach to dry eye disorders. The role of oral supplements with antioxidants and omega 3 fatty acids. Mol Vis. 2015 May 11;21:555-67.

19 Emwas AH. The strengths and weaknesses of NMR spectroscopy and mass spectrometry with particular focus on metabolomics research. Methods Mol Biol. 2015;1277:161-93.

20 Emwas AH, Roy R, McKay RT, Tenori L, Saccenti E, Gowda GAN, et al. NMR spectroscopy for metabolomics research. Metabolites. 2019 June 27;9(7):123..9

21 Wojtowicz JC, Butovich IA, McCulley JP. Historical brief on composition of human meibum lipids. Ocul Surf. 2009 July 01;7(3): $145-53$.

22 Geerling G, Baudouin C, Aragona P, Rolando M, Boboridis KG, Benítez-Del-Castillo JM, et al. Emerging strategies for the diagnosis and treatment of meibomian gland dysfunction: pProceedings of the OCEAN group meeting. Ocul Surf. 2017 April 01;15(2):179-92.

23 Borchman D, Ramakrishnan V, Henry C, Ramasubramanian A. Differences in mMeibum and tTear lLipid cComposition and cConformation. Cornea. 20192020 July Jan31;39(1): 122-8.

24 Nichols KK, Foulks GN, Bron AJ, Glasgow BJ, Dogru M, Tsubota K, et al. The international workshop on meibomian gland dysfunction: executive summary. Invest Ophthalmol Vis Sci. 2011 Mar 30;52(4):1922-9.

25 Schaumberg DA, Nichols JJ, Papas EB, Tong L, Uchino M, Nichols KK. The international workshop on meibomian gland dysfunction: report of the subcommittee on the epidemiology of, and associated risk factors for MGD. MGD. Invest .Ophthalmol .Vis .Sci.. 2011 Mar 30;52(4):1994-2005.

26 Schiffman RM, Christianson MD, Jacobsen G, Hirsch JD, Reis BL. Reliability and validity of the Ocular Surface Disease Index. Arch Ophthalmol. 2000 May;118(5):615-21.

27 Miller KL, Walt JG, Mink DR, Satram-Hoang S, Wilson SE, Perry HD, et al. Minimal clinically important difference for the ocular surface disease index. Arch Ophthalmol. 2010 January 01;128(1):94-101.

28 Tomlinson A, Bron AJ, Korb DR, Amano S, Paugh JR, Pearce EI, et al. The international workshop on meibomian gland dysfunction: report of the diagnosis subcommittee. Invest Ophthalmol Vis Sci. 2011 Mar 30;52(4):2006-49.

29 Butovich IA. Tear film lipids. Exp Eye Res. 2013 December 01;117:4-27.

30 Munoz-Hernandez AM, Galbis-Estrada C, Santos-Bueso E, Cuina-Sardina R, Diaz-Valle D, Gegundez-Fernandez JA, et al. Human tear metabolome. Arch. Soc. Esp. Oftalmol.. 2016 April 01;91(4):157-9.

31 Rossi C, Cicalini I, Cufaro MC, Agnifili L, Mastropasqua L, Lanuti P, et al. Multi-omics approach for studying tears in treatment-naive glaucoma patients. Int. J. Mol. Sci.. 2019 Aug 18;20(16):4029.
32 Yazdani M, Elgstøen KBP, Rootwelt H, Shahdadfar A, Utheim ØA, Utheim TP. Tear metabolomics in dry eye disease: a review. Int $\mathrm{J}$ Mol Sci. 2019 Aug 1;20(15):3755.

33 Nagele T, Furtauer L, Nagler M, Weiszmann J, Weckwerth W. A strategy for functional interpretation of metabolomic time series data in context of metabolic network information. Front. Mol. Biosci. 2016 Mar 7;3::36-6.

34 Grissa D, Pétéra M, Brandolini M, Napoli A, Comte B, Pujos-Guillot E. Feature selection methods for early predictive biomarker discovery using untargeted metabolomic data. Front Mol Biosci. 2016 July 08;3:30.

35 Rinaudo $\mathrm{P}$, Boudah S, Junot $\mathrm{C}$, Thévenot EA. biosignerBiosigner: a new method for the discovery of significant molecular signatures from omics data. Front Mol Biosci. 2016 Jun 21;3:26.

36 Nagele T. Linking metabolomics data to underlying metabolic regulation. Front $\mathrm{Mol}$ Biosci. 2014 November 06;1:22.

37 Emwas AH, Saccenti E, Gao X, McKay RT, Dos Santos VAPM, Roy R, et al. Recommended strategies for spectral processing and postprocessing of 1D (1)H-NMR data of biofluids with a particular focus on urine. Metabolomics. 2018;14(3):31-4.

38 Stapleton F, Alves M, Bunya VY, Jalbert I, Lekhanont K, Malet F, et al. TFOS DEWS II eEpidemiology rReport. Ocul Surf. 2017 Jul; 15(3):334-65.

39 Nakatsukasa M, Sotozono C, Shimbo K, Ono $\mathrm{N}$, Miyano H, Okano A, et al. Amino Acid acid profiles in human tear fluids analyzed by high-performance liquid chromatography and electrospray ionization tandem mass spectrometry. Am J Ophthalmol. 2011 May; 151(5):799-e1.

40 Rentka A, Koroskenyi K, Harsfalvi J, Szekanecz Z, Szucs G, Szodoray P, et al. Evaluation of commonly used tear sampling methods and their relevance in subsequent biochemical analysis. Ann Clin Biochem. 2017 Sep;54(5):521-9.

41 Ngo W, Chen J, Panthi S, Nichols KK, Nichols JJ. Comparison of collection methods for the measure of human meibum and tear film-derived lipids using mass spectrometry. Curr Eye Res. 2018 Oct;43(10):1244-52.

42 Choy CK, Cho P, Chung WY, Benzie IF. Water-soluble antioxidants in human tears: effect of the collection method. Invest Ophthalmol Vis Sci. 2001 December 01;42(13):31304.

43 Viant MR, Rosenblum ES, Tieerdema RS. NMR-based metabolomics: a powerful approach for characterizing the effects of environmental stressors on organism health. Environ Sci Technol. 2003 Nov 1;37(21):4982-9.

44 Wishart DS, Knox C, Guo AC, Eisner R, Young N, Bijaya Gautam B, et al. HMDB: a knowledge base for the human metabolome. Nucleic Acids Res. 2009;37(Database issue): D603-10. 
45 Chen L, Li J, Guo T, Ghosh S, Koh SK, Tian $\mathrm{D}$, et al. Global metabonomic and proteomic analysis of human conjunctival epithelial cells (IOBA-NHC) in response to hyperosmotic stress. J Proteome Res. 2015 September 04; 14(9):3982-95.

46 Di Venere M, Viglio S, Cagnone M, Bardoni A, Salvini R, Iadarola P. Advances in the analysis of “"less-conventional”" human body fluids: An an overview of the CE- and HPLC-MS applications in the years 2015--2017. Electrophoresis. 2018 Jan;39(1):160-78.

47 Aragona P, Rania L, Roszkowska AM, Spinella R, Postorino E, Puzzolo D, et al. Effects of amino acids enriched tears substitutes on the cornea of patients with dysfunctional tear syndrome. Acta Ophthalmol. 2013 September 01;91(6):e437.

48 Rusciano D, Roszkowska AM, Gagliano C, Pezzino S. Free amino acids: an innovative treatment for ocular surface disease. Eur J Pharmacol. 2016 Sep 15;787:9-19.

49 Pieragostino D, D"Alessandro $\mathrm{M}$, di Ioia $\mathrm{M}$, Di Ilio C, Sacchetta P, Del Boccio P. Unraveling the molecular repertoire of tears as a source of biomarkers: beyond ocular diseases. Proteomics Clin Appl. 2015 Feb;9(1-2):16986.

50 Geiger R, Rieckmann JC, Wolf T, Basso C, Feng Y, Fuhrer T, et al. L-Arginine Modulates modulates $\mathrm{T}$ cell metabolism and enhances survival and anti-tumor activity. Cell. 2016 October 20;167(3):829-e42.e13.

51 Shamsheer RP, Arunachalam C. A clinical study of meibomian gland dysfunction in patients with diabetes. Middle East. Middle East AAfr. J. Ophthalmol. 2015;22(4):462-6.

52 Baca JT, Finegold DN, Asher SA. Tear glucose analysis for the noninvasive detection and monitoring of diabetes mellitus. Ocul Surf. 2007 October 01;5(4):280-93.

53 van Haeringen NJ, Glasius E. Cholesterol in human tear fluid. Exp Eye Res. 1975 Mar; 20(3):271-4.
54 Wollensak G, Mur E, Mayr A, Baier G, Göttinger W, Stöffler G. Effective methods for the investigation of human tear film proteins and lipids. Graefes Arch Clin Exp Ophthalmol. 1990;228(1):78-82.

55 Ham BM, Jacob JT, Keese MM, Cole RB. Identification, quantification and comparison of major non-polar lipids in normal and dry eye tear lipidomes by electrospray tandem mass spectrometry. J Mass Spectrom. 2004 November 01;39(11):1321-36.

56 Ham BM, Jacob JT, Cole RB. MALDI-TOF MS of phosphorylated lipids in biological fluids using immobilized metal affinity chromatography and a solid ionic crystal matrix. Anal Chem. 2005 Jul 15;77(14):4439-47.

57 Butovich IA, Uchiyama E, Di Pascuale MA, McCulley JP. Liquid chromatography-mass spectrometric analysis of lipids present in human meibomian gland secretions. Lipids. 2007 Aug;42(8):765-76.

58 Butovich IA, Millar TJ, Ham BM. Understanding and analyzing meibomian lipids--: a review. Curr Eye Res. 2008 May;33(5):40520.

59 Borchman D, Foulks GN, Yappert MC, Ho DV. Temperature-induced conformational changes in human tearlipids hydrocarbon chains. Biopolymers. 2007;87(2-3):124-33.

60 Borchman D, Foulks GN, Yappert MC, Milliner SE. Differences in human meibum lipid composition with meibomian gland dysfunction using NMR and principal component analysis. Invest Ophthalmol Vis Sci. 2012 Jan 25;53(1):337-47.

61 Butovich IA, McMahon A, Wojtowicz JC, Lin F, Mancini R, Itani K. Dissecting lipid metabolism in meibomian glands of humans and mice: An an integrative study reveals a network of metabolic reactions not duplicated in other tissues. Biochim Biophys Acta. 2016 Jun;1861(6):538-53.

62 Dahlhoff M, Camera E, Schäfer M, Emrich D, Riethmacher D, Foster A, et al. Sebaceous lipids are essential for water repulsion, protection against UVB-induced apoptosis and ocular integrity in mice. Development. 2016 May 15;143(10):1823-31.
63 Baudouin C. [A new approach for better comprehension of diseases of the ocular surface]. J Fr Ophtalmol. 2007 Mar;30(3):239-46.

64 Sweeney DF, Millar TJ, Raju SR. Tear film stability: a review. Exp Eye Res. 2013 Dec;117: 28-38.

65 Garcia-Posadas L, Contreras-Ruiz L, LopezGarcia A, Villaron Alvarez S, Maldonado MJ, Diebold Y. Hyaluronan receptors in the human ocular surface: a descriptive and comparative study of RHAMM and CD44 in tissues, cell lines and freshly collected samples. Histochem. Cell Biol.. 2012 February 01; 137(2):165-76.

66 Lam SM, Tong L, Duan X, Acharya UR, Tan $\mathrm{JH}$, Petznick A, et al. Longitudinal changes in tear fluid lipidome brought about by eyelidwarming treatment in a cohort of meibomian gland dysfunction. J Lipid Res. 2014 Sep; 55(9):1959-69.

$67 \mathrm{Hu}$ X, Lyu H, Jiang X, Li X. [Changes in human meibum with meibomian gland dysfunction]. Zhonghua Yan Ke Za Zhi. 2015 Mar;51(3):225-8.

68 Villani E, Baudouin C, Efron N, Hamrah P, Kojima T, Patel SV, et al. In vivo confocal microscopy of the ocular surface: from bench to bedside. Curr Eye Res. 2014 Mar;39(3):21331.

69 Villani E, Canton V, Magnani F, Viola F, Nucci P, Ratiglia R. The aging Meibomian gland: an in vivo confocal study. Invest Ophthalmol Vis Sci. 2013 Jul 16;54(7):4735-40.

70 Napoli PE, Coronella F, Satta GM, Iovino C, Sanna R, Fossarello M. A simple novel technique of infrared meibography by means of spectral-domain optical coherence tomography: a cross-sectional clinical study. PLoS One. 2016 Oct 31;11(10):e0165558.

71 Chen L, Gao Y, Wang LZ, Cheung N, Tan GSW, Cheung GCM, et al. Recent advances in the applications of metabolomics in eye research. Anal Chim Acta. 2018;1037:28-40. 